\title{
The pion holographic wavefunction with dynamical spin effects
}

\section{Mohammad Ahmady ${ }^{* \dagger}$}

Department of Physics, Mount Allison University, Sackville, New Brunswick, Canada E4L 1E6

E-mail: mahmady@mta.ca

\section{Farrukh Chishtie}

Department of Physics and Astronomy, The University of Western Ontario, London, Canada N6A3K7

E-mail: fchishti@uwo.ca

\section{Ruben Sandapen}

Department of Physics, Acadia University, Wolfville, Nova-Scotia, Canada, B4P 2R6 and Department of Physics, Mount Allison University, Sackville, New Brunswick, Canada, E4L 1E6 E-mail: ruben. sandapen@acadiau.ca

We report that the inclusion of dynamical spin effects in the pion holographic light-front wavefunction leads to a remarkable improvement in describing pion observables (pion mean charge radius, decay constant, spacelike electromagnetic form factor) without the need to invoke higher Fock state contributions and/or a special AdS/QCD mass scale for the pion.

XVII International Conference on Hadron Spectroscopy and Structure

25-29 September, 2017

University of Salamanca, Salamanca, Spain

\footnotetext{
${ }^{*}$ Speaker.

${ }^{\dagger}$ This research is supported by NSERC Discovery Grants SAPIN-2017-00033 (M.A) and SAPIN-2017-00031 (R.S).
} 


\section{Introduction}

For massless quarks, the meson holographic light-front wavefunctions (LFWFs) can be written as:[1]

$$
\Psi_{n L}(\zeta, x, \phi)=e^{i L \phi} \sqrt{x(1-x)}(2 \pi)^{-1 / 2} \kappa^{1+L} \sqrt{\frac{2 n !}{(n+L) !}} \zeta^{L} \exp \left(\frac{-\kappa^{2} \zeta^{2}}{2}\right) L_{n}^{L}\left(x^{2} \zeta^{2}\right)
$$

where $x$ is the light-front (LF) momentum fraction carried by the quark and $\zeta^{2}=x(1-x) b^{2}$ where $b$ is the transverse separation between quark and antiquark. The corresponding meson mass $M$ is:

$$
M^{2}=4 \kappa^{2}\left(n+L+\frac{S}{2}\right)
$$

with $L(S)$ being the orbital (spin) angular momentum, and $\kappa$ and $n$, similar to (1.1), are the AdS/QCD scale and the quantum number, respectively. We note that the lowest lying meson ( $n=L=S=0$ ), i.e. pion, is predicted to be massless in the chiral limit, as expected.

Non-zero quark masses is usually implemented following the prescription of Brodsky and de Téramond [2]. For the ground state pion, this leads to

$$
\Psi^{\pi}\left(x, \zeta^{2}\right)=\mathscr{N} \sqrt{x(1-x)} \exp \left[-\frac{\kappa^{2} \zeta^{2}}{2}\right] \exp \left[-\frac{m_{f}^{2}}{2 \kappa^{2} x(1-x)}\right]
$$

where the normalization constant $\mathscr{N}$ is fixed by requiring that

$$
\int \mathrm{d}^{2} \mathbf{b d} x\left|\Psi^{\pi}\left(x, \zeta^{2}\right)\right|^{2}=P_{q \bar{q}}
$$

with $P_{q \bar{q}}$ being the probability that the meson consists of the leading quark-antiquark Fock state.

\section{Model parameters and pion data}

A AdS/QCD scale $\kappa=520-590 \mathrm{MeV}$ is required to fit the experimentally measured Regge slopes for pseudoscalar and vector mesons [1] as well as baryons [3]. Furthermore, a fit to the HERA data on diffractive $\rho$ electroproduction, with $m_{u / d}=140 \mathrm{MeV}$, gives $\kappa=560 \mathrm{MeV}$ [4] and using $\kappa=550 \mathrm{MeV}$ (with $m_{u / d}\left[m_{s}\right]=46[140] \mathrm{MeV}$ ) leads to a good simultaneous description of the HERA data on diffractive $\rho$ and $\phi$ electroproduction [5]. Therefore, one may conclude that a universal AdS/QCD scale $\kappa \sim 550 \mathrm{MeV}$ is applicable to all hadronic bound states. However, pion is a notable exception:

- for massless quarks, much lower values of $\kappa$ were required to fit the pion data, $\kappa=375 \mathrm{MeV}$ to fit the pion EM form factor data [6] and $\kappa=432 \mathrm{MeV}$ (with $P_{q \bar{q}}=0.5$ ) to fit the photonto-pion transition form factor data simultaneously at large $Q^{2}$ and $Q^{2}=0$ (the latter is fixed by the $\pi^{0} \rightarrow \gamma \gamma$ decay width) [7].

- with $m_{u / d}=330 \mathrm{MeV}$, a higher value of $\kappa=787 \mathrm{MeV}$ is needed along with $P_{q \bar{q}}=0.279$, implying an important contribution of higher Fock states in the pion Ref. [8]. 
- with $m_{u / d}=420 \mathrm{MeV}$, a universal AdS/QCD scale $\kappa=550 \mathrm{MeV}$ can be used for pion only if $P_{q \bar{q}}=0.6$ [9].

All these previous studies seem to indicate that a special treatment is required at least for the pion either by using a distinct AdS/QCD scale $\kappa$ or/and relaxing the normalization condition on the holographic wavefunction, i.e. invoking higher Fock states contributions. However, one should note that in the above papers the pion observables are predicted using the holographic wavefunction, given by Eq. (1.3), with the quark helicity dependence is always assumed to decouple from the dynamics:

$$
\Psi_{h \bar{h}}^{\pi[\mathrm{o}]}(x, \mathbf{b})=\frac{1}{\sqrt{2}} h \delta_{-h \bar{h}} \Psi^{\pi}\left(x, \zeta^{2}\right)
$$

In Ref [10], we show that it is possible to achieve a better description of the pion observables by using a universal AdS/QCD scale $\kappa$ and without the need to invoke higher Fock state contributions. We do so by taking into account dynamical spin effects in the holographic pion wavefunction, i.e. we use a momentum-dependent helicity wavefunction.

\section{Dynamical spin effects}

To restore the helicity dependence of the holographic wavefunction, we assume that

$$
\Psi(x, \mathbf{k}) \rightarrow \Psi_{h \bar{h}}(x, \mathbf{k})=S_{h \bar{h}}(x, \mathbf{k}) \Psi(x, \mathbf{k})
$$

where $S_{h \bar{h}}(x, \mathbf{k})$ corresponds to the helicity wavefunction for a point-like meson- $q \bar{q}$ coupling. For vector mesons, the helicity wavefunction is therefore similar to that of the point-like photon- $q \bar{q}$ coupling, i.e.

$$
S_{h \bar{h}}^{V}(x, \mathbf{k})=\frac{\bar{v}_{\bar{h}}\left((1-x) P^{+},-\mathbf{k}\right)}{\sqrt{(1-x)}}\left[\gamma \cdot \varepsilon_{V}\right] \frac{u_{h}\left(x P^{+}, \mathbf{k}\right)}{\sqrt{x}}
$$

where $\varepsilon_{V}^{\mu}$ is the polarization vector of the vector meson. For the pseudoscalar pion, we replace $\gamma \cdot \varepsilon_{V}$ in Eq. (3.2) by (scalar function) $\times \gamma^{5}$ where the most general, dimensionally homogeneous, scalar function that can be constructed using the pion's momentum is $A(P \cdot \gamma)+B \sqrt{P \cdot P}$ with $A$ and $B$ being arbitrary constants. Hence

$$
S_{h \bar{h}}^{\pi}(x, \mathbf{k})=\frac{\overline{\bar{\nu}}_{\bar{h}}\left((1-x) P^{+},-\mathbf{k}\right)}{\sqrt{1-x}}\left[\left(A \not P+B M_{\pi}\right) \gamma^{5}\right] \frac{u_{h}\left(x P^{+}, \mathbf{k}\right)}{\sqrt{x}} .
$$

Using the light-front spinors [11], we obtain

$$
S_{h \bar{h}}^{\pi}(x, \mathbf{k})=\left\{A M_{\pi}^{2}+B\left(\frac{m_{f} M_{\pi}}{x(1-x)}\right)\right\}(2 h) \delta_{-h \bar{h}}+B\left(\frac{M_{\pi} k e^{i(2 h) \theta_{k}}}{x(1-x)}\right) \delta_{h \bar{h}}
$$

with $\mathbf{k}=k e^{i \theta_{k}}$. We note that if we take $B=0$, the helicity wavefunction becomes momentumindependent:

$$
S_{h \bar{h}}^{\pi}(x, \mathbf{k}) \rightarrow S_{h \bar{h}}^{\pi}=\frac{1}{\sqrt{2}}(2 h) \delta_{-h \bar{h}}
$$

and so dynamical spin effects are included only if $B \neq 0$. Table 1 shows our predictions of the pion charge radius with the original $(B=0)$ and dynamical spin wavefunctions. As can be seen, a 


\begin{tabular}{|c|c|}
\hline & $\sqrt{\left\langle r_{\pi}^{2}\right\rangle}[\mathrm{fm}]$ \\
\hline Original & 0.544 \\
\hline Spin-improved $(A=0, B=1)$ & 0.683 \\
\hline Spin-improved $(A=1, B=1)$ & 0.673 \\
\hline Experiment $[12]$ & $0.672 \pm 0.008$ \\
\hline
\end{tabular}

Table 1: Our predictions for the pion radius using the holographic wavefunction with $\kappa=523 \mathrm{MeV}$ and $m_{u / d}=330 \mathrm{MeV}$. The datum is from PDG 2014 [12].

\begin{tabular}{|c|c|}
\hline & $f_{\pi}[\mathrm{MeV}]$ \\
\hline Original & 161 \\
\hline Spin-improved $(A=0, B=1)$ & 135 \\
\hline Spin-improved $(A=1, B=1)$ & 138 \\
\hline Experiment $[12]$ & $130.4 \pm 0.04 \pm 0.2$ \\
\hline
\end{tabular}

Table 2: Our predictions for the pion decay constant using the holographic wavefunction with $\kappa=523 \mathrm{MeV}$ and $m_{u / d}=330 \mathrm{MeV}$. The datum is from PDG 2014 [12].

much better agreement with the experimental data is achieved with the spin-improved holographic wavefunctions.

Our predictions for the pion decay constant are shown in Table 2. As can be seen, also for this observable we achieve a much better agreement with the data when the spin-improved holographic wavefunctions is used although we still somewhat overestimate the measured value. This could perhaps be attributed to the fact that perturbative corrections are not included in the holographic pion wavefunction.

Figure 1 illustrates our predictions for the EM form factor using the original (dotted-orange curve) and the dynamical spin-improved (continuous-red and dashed-blue curve) as compared with the data from CERN [13], CEA [14], Cornell [15, 16, 17], Jlab [18, 19] and CLEO [20, 21]. As can be seen, the agreement with data is very much improved with the spin-improved holographic wavefunctions. In fact, we achieve excellent agreement with data from the lowest $Q^{2}$ datum to $Q^{2} \approx 7 \mathrm{GeV}^{2}$. For $Q^{2}>7 \mathrm{GeV}^{2}$, our predictions with the original and the spin-improved holographic wavefunctions coincide and they both undershoot the precise CLEO data [20]. This is the short-distance regime where perturbative corrections, not taken into account in the purely nonperturbative holographic wavefunction, become important. It is worth highlighting that agreement with the precise data in the non-perturbative region, $Q^{2} \leq 1 \mathrm{GeV}^{2}$, is excellent with our higher twist spin-improved holographic wavefunctions.

\section{4. conclusion}

We have shown that if dynamical spin effects are taken into account, the $\pi$-mesons can be treated like other mesons in light-front holography. 


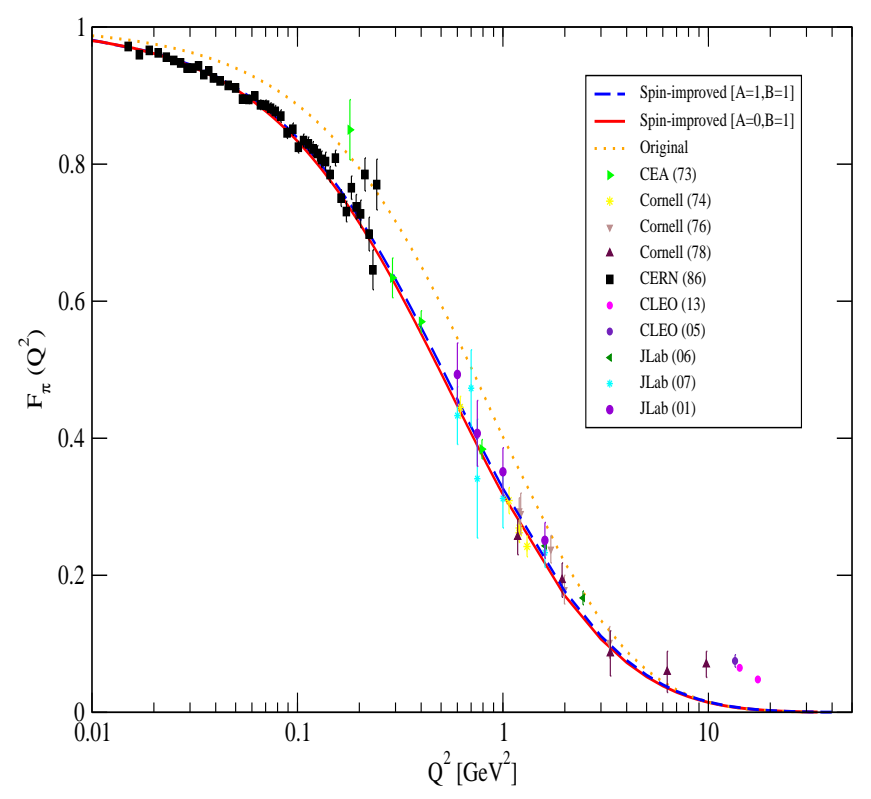

Figure 1: Our predictions for the pion EM form factor. Dotted-orange: original. Continuous-red curve: spin-improved $(A=0, B=1)$. Dashed-blue curve: spin-improved $(A=1, B=1)$. All theory curves generated with $\kappa=523 \mathrm{MeV}$ and $m_{f}=330 \mathrm{MeV}$. Data from [13, 15, 16, 17, 18, 19, 20, 21].

\section{5. acknowledgment}

MA thanks the organizers of Hadron 2017 for a successful conference in Salamanca.

\section{References}

[1] S. J. Brodsky, G. F. de Teramond, H. G. Dosch, and J. Erlich, Light-Front Holographic QCD and Emerging Confinement, Phys. Rept. 584 (2015) 1-105, [1407.8131].

[2] S. J. Brodsky and G. F. de Teramond, AdS/CFT and Light-Front QCD, Subnucl. Ser. 45 (2009) 139-183, [0802.0514].

[3] S. J. Brodsky, G. F. de Teramond, H. G. Dosch, and C. Lorce, Meson/Baryon/Tetraquark Supersymmetry from Superconformal Algebra and Light-Front Holography, Int. J. Mod. Phys. A31 (2016), no. 19 1630029, [1606.04638].

[4] J. R. Forshaw and R. Sandapen, An AdS/QCD holographic wavefunction for the $\rho$ meson and diffractive $\rho$ meson electroproduction, Phys. Rev. Lett. 109 (2012) 081601, [1203 . 6088].

[5] M. Ahmady, R. Sandapen, and N. Sharma, Diffractive $\rho$ and $\phi$ production at HERA using an AdS/QCD holographic light-front meson wavefunction, 1605.07665.

[6] S. J. Brodsky and G. F. de Teramond, Light-Front Dynamics and AdS/QCD Correspondence: The Pion Form Factor in the Space-and Time-Like Regions, Phys. Rev. D77 (2008) 056007, [0707.3859]. 
[7] S. J. Brodsky, F.-G. Cao, and G. F. de Teramond, Meson Transition Form Factors in Light-Front Holographic QCD, Phys. Rev. D84 (2011) 075012, [1105.3999].

[8] A. Vega, I. Schmidt, T. Branz, T. Gutsche, and V. E. Lyubovitskij, Meson wave function from holographic models, Phys. Rev. D80 (2009) 055014, [0 906 . 1220].

[9] T. Branz, T. Gutsche, V. E. Lyubovitskij, I. Schmidt, and A. Vega, Light and heavy mesons in a soft-wall holographic approach, Phys. Rev. D82 (2010) 074022, [1 008 . 0268 ].

[10] M. Ahmady, F. Chishtie, and R. Sandapen, Spin effects in the pion holographic light-front wavefunction, Phys. Rev. D95 (2017), no. 7 074008, [1609.07024].

[11] G. P. Lepage and S. J. Brodsky, Exclusive Processes in Perturbative Quantum Chromodynamics, Phys. Rev. D22 (1980) 2157.

[12] Particle Data Group Collaboration, K. A. Olive et al., Review of Particle Physics, Chin. Phys. C38 (2014) 090001.

[13] NA7 Collaboration, S. R. Amendolia et al. Nucl. Phys. B277 (1986) 168.

[14] C. N. Brown et al. Phys. Rev. D8 (1973) 92-135.

[15] C. J. Bebek et al. Phys. Rev. D9 (1974) 1229-1242.

[16] C. J. Bebek et al. Phys. Rev. D13 (1976) 25.

[17] C. J. Bebek et al. Phys. Rev. D17 (1978) 1693.

[18] J. V. et al (The Jefferson Lab F $\pi$ Collaboration) Phys. Rev. Lett. 86 (2001) 1713, [hep-ph/0602252].

[19] T. H. et al (The Jefferson Lab F $\pi$ Collaboration) Phys. Rev. Lett. 97 (2006) 192001, [nucl-ex/0607005].

[20] T. K. P. et al (CLEO collaboration) Phys. Rev. Lett. 95 (2005) 261803, [hep-ex/ 0510005 ].

[21] K. K. Seth, S. Dobbs, Z. Metreveli, A. Tomaradze, T. Xiao, and G. Bonvicini Phys. Rev. Lett. 110 (2013), no. 2 022002, [1210.1596]. 